\title{
Nonfragile Integral-Based Event-Triggered Control of Uncertain Cyber-Physical Systems under Cyber-Attacks
}

\author{
Shen Yan $\mathbb{D}^{1},{ }^{1}$ Sing Kiong Nguang $\mathbb{D}^{2},{ }^{2}$ and Liruo Zhang ${ }^{2}$ \\ ${ }^{1}$ College of Mechanical \& Electronic Engineering, Nanjing Forestry University, Nanjing 210037, China \\ ${ }^{2}$ Department of Electrical, Computer and Software Engineering, The University of Auckland, Auckland 1142, New Zealand
}

Correspondence should be addressed to Shen Yan; yanshenzdh@gmail.com

Received 29 June 2019; Revised 23 August 2019; Accepted 14 September 2019; Published 3 November 2019

Academic Editor: Xinggang Yan

Copyright (c) 2019 Shen Yan et al. This is an open access article distributed under the Creative Commons Attribution License, which permits unrestricted use, distribution, and reproduction in any medium, provided the original work is properly cited.

This article studies the problem of nonfragile integral-based event-triggered control for uncertain cyber-physical systems under cyber-attacks. An integral-based event-triggered scheme is proposed to reduce the data transmissions and save the limited network resources. The triggering condition is related to the mean of system state over a finite time interval instead of instant system state. Random cyber-attacks in a communication channel are taken into account and described by a stochastic variable subject to Bernoulli distribution. A novel Lyapunov-Krasovskii functional based on Legendre polynomials is constructed, and the Bessel-Legendre inequality technique is employed to handle the integral term induced by the integral-based event-triggered scheme. Resorting to these treatments, sufficient conditions are established via a set of linear matrix inequalities to guarantee the asymptotic mean-square stability of the closed-loop system. Finally, a numerical example shows that the presented method is effective.

\section{Introduction}

Cyber-physical systems (CPSs) are systems where computational and physical resources are tightly integrated via a shared network. They have been applied in industrial fields, such as medical devices, chemical process industries, and transportation systems [1-3]. Security is a critical issue in CPSs, as a great deal of communication signals are exchanged over the network channel that is open to potential cyber-attacks. Cyber-attacks, introduced by external attackers, could cause negative impact on the communication channels and deteriorate system stability performance. Thus, it has attracted much attention to study the control and filtering problems for CPSs under the threats of network attacks [4-7]. The two representative kinds of cyber-attacks are, namely, deception attacks and denial-of-service (DoS) attacks. The deception attacks aim at injecting deceitful information into the transmitted signal, and the DoS attacks could block or break the communication link among system nodes. Some recent results on the study of cyber-attacks are reported in $[8,9]$. The distributed recursive filtering problem of stochastic systems with time delays and deception attacks is investigated in [8]. The finite-horizon tracking control issue of a stochastic system subject to hybrid attacks is developed in [9], where both the stochastic deception attacks and stochastic DoS attacks are taken into consideration.

Note that, in the above results, communication signals are transmitted through the network without considering some practical constraints, such as limited network bandwidth and computation burden. Some effective manners such as event-triggered scheme (ETS) [10], signal quantization [11], and real-time scheduling [12,13] have been proposed to save communication resources and reduce computation burden. Recently, ETS has gained growing attention and interests in existing research studies [14-23] for its simplicity of execution and effectiveness of reducing data transmissions. Due to this fact, the idea of ETS is introduced into security control for CPSs and has produced many interesting results [24-29]. To be specific, in [24], a resilient $H_{\infty}$ load frequency controller is designed to stabilize the multiarea power systems with cyber-attacks. The issue of event-triggered controller design for networked 
systems under the resilient event-triggered mechanism and periodic DoS jamming attacks is developed in [25]. For multiagent systems with deception attacks, [26] is concerned with the event-triggered output consensus control issue. Zha et al. [27] investigate the design of decentralized eventtriggered $H_{\infty}$ controller of delayed neural networks under deception attacks. Liu et al. [28] address the distributed event-triggered control issue for networked control systems with stochastic cyber-attacks, and a decentralized triggering strategy is utilized to release the network communication burden. In [29], the resilient load frequency control problem for multiarea power systems with cyber-attacks is investigated by using an event-triggered communication mechanism. In these studies, only the instant system information, such as current system state and the last triggered signal, is applied to design the ETS conditions. In practical systems, the system state could have abrupt fluctuations over some time interval incurred by external disturbances, system uncertainties, or environment noises. If such state with abrupt fluctuations is triggered to controller, it is negative for stabilizing the system performance. In [30], the idea of introducing the accumulation error information or system dynamics over a finite time interval into the design of triggering rule is proposed. Considering systems with stochastic measurement noises, an integral-based ETS utilizing the average state over a finite time interval is proposed to reduce the effect of measurement noises in [31], where an approximation approach is used to deal with the integral triggering condition. This method to treat the integral term will result in approximation error, which needs further investigation to exclude such approximation error and obtain less conservative results. In addition, the controllers in these works are assumed to be implemented perfectly. However, uncertainties caused by execution errors or unknown noises are usually observed in engineerings, which may lead to system performance deterioration. To conquer this problem, Sakthivel et al. [32] handle the uncertainties by norm-bounded variations to design the dissipative analysis-based nonfragile controller for network-based singular systems with ETS. In [33], the issue of nonfragile event-triggered $H_{\infty}$ control of linear systems under unreliable communication links is addressed. For linear systems against actuator saturation and disturbances, Liu and Yang [34] study the design of nonfragile dynamic output feedback controller via an event-triggered communication scheme. It is noted that the cyber-attacks are not considered in communication channels, and the mean of system state is not taken into account for ETS design in [32-34]. To our best knowledge, few results have been investigated for integralbased event-triggered control problem of uncertain systems with cyber-attacks and gain uncertainties, which inspire the current study.

This paper focuses on the co-design of integral-based ETS and nonfragile controller for uncertain CPSs with cyber-attacks and gain uncertainties. The main contributions of this paper are summarized as follows:

(1) An integral-based ETS that utilizes the mean of system state over a fixed time interval is proposed to reduce the wastage of limited network resources. It covers the conventional ETS [10] as a special case as the fixed time interval approaches to zero and can further reduce unnecessary data transmissions.

(2) The closed-loop system with the integral-based ETS, cyber-attacks, and gain variations is established as a distributed delay system, in which a Bernoulli variable is adopted to describe the stochastic cyber-attacks. A novel augmented Lyapunov-Krasovskii functional (LKF) based on Legendre polynomials is constructed. With the help of the novel LKF and Bessel-Legendre inequality, the integral term induced by integralbased ETS is handled without the approximation error in [31].

The organization of this paper is given as follows. Section 2 presents the problem statement and some preliminaries. The main results of stability analysis and control synthesis are derived in Section 3. To show the effectiveness of the proposed approach, a numerical example is given in Section 4. Some conclusions and future research topics are shown in Section 5.

1.1. Notation. In this paper, $\mathbb{R}^{n}$ and $\mathbb{R}^{n \times m}$ denote the $n$ dimensional Euclidean space and the set of all $n \times m$ real matrices, respectively. $\|\cdot\|$ is the Euclidean norm in $\mathbb{R}^{n}$. $\mathbb{E}\{\cdot\}$ means the mathematical expectation. The notation $X>0$ $(<0)$ represents that $X$ is symmetric and positive (negative) definite. The superscript " $T$ " stands for the transpose of a vector or matrix. $X^{\perp}$ denotes the kernel of $X . H e(X)$ equals to $X^{T}+X$. The notation $\left(\begin{array}{l}k \\ j\end{array}\right)$ means the binomial coefficients given by $k ! /(k-j) ! j ! . \otimes$ refers to the Kronecker product. $\mathbb{N}$ is the set of nonnegative integers.

\section{Preliminaries}

Consider the following uncertain continuous-time system as

$$
\dot{x}(t)=(A+\Delta A(t)) x(t)+B u(t),
$$

where $x(t) \in \mathbb{R}^{n}$ is the system state, $u(t) \in \mathbb{R}^{m}$ is system input, $A$ and $B$ are known system matrices, and $\Delta A(t)=\varsigma(t) \widehat{A}$, where $\varsigma(t)$ is a stochastic variable with expectation $\bar{\zeta}$ and variance $\widetilde{\zeta}^{2}$ and $\widehat{A}$ means the deviation from the nominal model. Different from the commonly used uncertainty model $\Delta A(t)=D F(t) E$ with exactly known bounds, we focus on the statistic of the uncertainty deviating from the nominal part.

Since the network bandwidth is limited, unnecessary data transmission will lead to the wastage of limited network resources. In order to decrease communication frequency, an integral-based event-triggered scheme, drawn in Figure 1, is presented as

$$
s_{k+1}=\min _{s}\left\{s \geq s_{k} \mid e^{T}(s) \Phi e(s) \geq \delta \widehat{x}^{T}\left(s_{k}\right) \Phi \widehat{x}\left(s_{k}\right)\right\}
$$

where $\delta \in[0,1) ; \quad e(s)=1 / \tau \int_{s-\tau}^{s} x(v) \mathrm{d} v-\widehat{x}\left(s_{k}\right), \quad 1 / \tau \int_{s-\tau}^{s}$ $x(v) \mathrm{d} v$ is the mean of system state; $\widehat{x}\left(s_{k}\right)=1 / \tau \int_{s_{k}-\tau}^{s_{k}} x(v) \mathrm{d} v$ and $\widehat{x}\left(s_{k+1}\right)=1 / \tau \int_{s_{k+1}-\tau}^{s_{k+1}} x(v) \mathrm{d} v$, the mean of the current and 


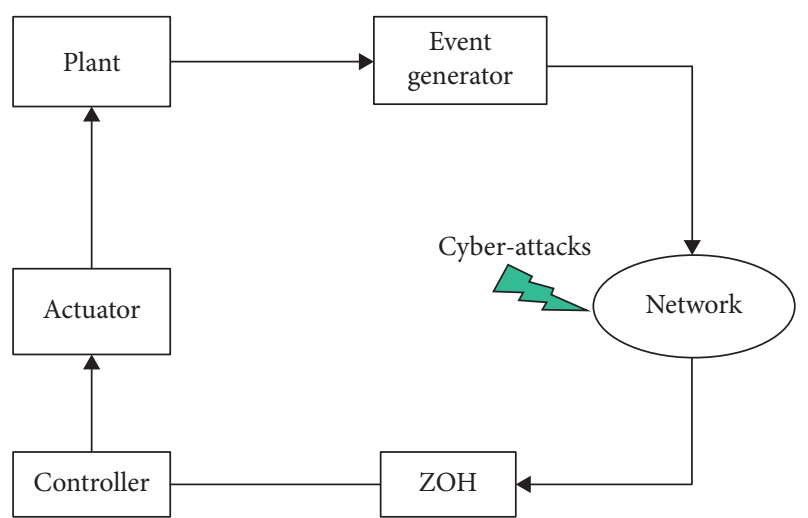

FIGURE 1: Framework of event-triggered control for CPSs against cyber-attacks.

next triggered control signal, respectively; $\tau$ is the length of the mean of system state over $[s-\tau, s)$; and $\Phi$ is the positive weighting matrix.

Remark 1. The proposed triggering condition (2) takes into account the mean of system state over $[s-\tau, s)$, formulated as $1 / \tau \int_{s-\tau}^{s} x(v) \mathrm{d} v$, instead of the instant system state $x(s)$. The triggering condition is then formed by integration, which motivates the name of integral-based ETS.

Remark 2. When $\tau \longrightarrow 0, e(s)=\int_{s-\tau}^{s}(1 / \tau) x(v) \mathrm{d} v-\widehat{x}\left(s_{k}\right)$ is equivalent to $x(s)-x\left(s_{k}\right)$. Then, the triggering mechanism (2) is rewritten as

$$
\begin{aligned}
s_{k+1}= & \min _{s}\left\{s \geq s_{k} \mid\left(x(s)-x\left(s_{k}\right)\right)^{T}\right. \\
& \left.\cdot \Phi\left(x(s)-x\left(s_{k}\right)\right) \geq \delta x\left(s_{k}\right)^{T} \Phi x\left(s_{k}\right)\right\},
\end{aligned}
$$

which reduces to the normal ETS proposed in [10].

In real systems, the network transmission delay is a critical problem, which may cause system performance degradation. It is usually modeled by constant delay and time-varying delay. Here, the constant network transmission delay is considered and denoted by $\tau_{1}$, which meets

$$
t_{k}=s_{k}+\tau_{1}<s_{k+1}+\tau_{1}=t_{k+1}, \quad k \in \mathbb{N} .
$$

Therefore, combining the transmission delay and zero-order holder $(\mathrm{ZOH})$ with the triggering condition (2), it gives that

$$
\varepsilon^{T}(t) \Phi \mathcal{E}(t)<\delta \widehat{x}^{T}\left(s_{k}\right) \Phi \hat{x}\left(s_{k}\right), \quad t \in\left[t_{k}, t_{k+1}\right),
$$

where $\varepsilon(t) \triangleq 1 / \tau \int_{t-\tau_{2}}^{t-\tau_{1}} x(v) \mathrm{d} v-\widehat{x}\left(s_{k}\right), \tau_{2}=\tau_{1}+\tau, \varepsilon(t)$ is the error term, and $1 / \tau \int_{t-\tau_{2}}^{t-\tau_{1}} x(v) \mathrm{d} v$ will be used to represent the control signal $\hat{x}\left(s_{k}\right)$ to establish the closed-loop system.

It is noted that the data communication over the network channel between event generator and controller is easy to be attacked by hackers. The random cyber-attacks on communication signals could lead to inaccurate control operations. Moreover, taking into account the controller gain variations, the control input reflecting the gain variations and cyber-attacks is modeled below:

$$
\begin{aligned}
u(t)= & \alpha\left(s_{k}\right)(K+\Delta K) \hat{x}\left(s_{k}\right) \\
& \left.+\left(1-\alpha\left(s_{k}\right)\right)(K+\Delta K)\right) f\left(\widehat{x}\left(s_{k}\right)\right), \quad t \in\left[t_{k}, t_{k+1}\right),
\end{aligned}
$$

where $\alpha\left(s_{k}\right) \in\{0,1\}$ is a Bernoulli variable satisfying the probability: $\operatorname{Prob}\left\{\alpha\left(s_{k}\right)=1\right\}=\bar{\alpha}$ and $\operatorname{Prob}\left\{\alpha\left(s_{k}\right)=0\right\}=$ $1-\bar{\alpha}$, respectively; the triggered data is attacked by external hacker with the cyber-attack function $f\left(\widehat{x}\left(s_{k}\right)\right)$ for $\alpha\left(s_{k}\right)=0 ; K$ is the controller gain to be designed; and $\Delta K=$ $E_{a} G(t) E_{b}$ is the gain uncertainty satisfying the normbounded condition $G^{T}(t) G(t) \leq I$.

Remark 3. The impact of cyber-attacks on the transmitted data is described by a stochastic variable $\alpha\left(s_{k}\right)$ obeying Bernoulli distribution. When $\alpha\left(s_{k}\right)=1$, it represents the triggered data sent to the controller successfully. For $\alpha\left(s_{k}\right)=0$, the triggered data is attacked by deception signals as transmitting over the network channel.

Remark 4. In this paper, the controller is designed considering only the stochastic occurring deception attacks whose purpose is to degrade the system performance and stability by corrupting or saturating the control inputs.

Therefore, substituting (6) and $\varepsilon(t)$ defined in (5) into (1), the closed-loop system is modeled as a system with a distributed input delay:

$$
\begin{aligned}
\dot{x}(t)= & (A+\Delta A(t)) x(t)+\alpha\left(s_{k}\right) B(K+\Delta K) \\
& \cdot\left(\frac{1}{\tau} \int_{t-\tau_{2}}^{t-\tau_{1}} x(v) \mathrm{d} v-\varepsilon(t)\right) \\
& +\left(1-\alpha\left(s_{k}\right)\right) B(K+\Delta K) f\left(\hat{x}\left(s_{k}\right)\right) .
\end{aligned}
$$

This aim of this paper is to design nonfragile state feedback controller (6) such that the closed-loop system (2) under the integral-based ETS and cyber-attacks is asymptotically stable.

The following assumption and technical lemmas will be utilized in achieving the main results.

Assumption 1. The cyber-attack function $f(\cdot)$ in (6), for $i=1,2, \ldots, n$, is assumed to meet the sector-bounded condition

$$
\left(f(x)-F_{1} x\right)^{T}\left(f(x)-F_{2} x\right) \leq 0,
$$

where $F_{1}$ and $F_{2}$ are two real constant matrices and satisfy $F_{2}-F_{1} \geq 0$.

Remark 5. The cyber-attack function $f(\cdot)$ that satisfies Assumption 1 can be signal saturation $f(x(t))=\operatorname{sat}(x(t))$, time-varying percentage function $f(x(t))=(I+\Delta(t)) x(t)$, where $\Delta(t)$ is bounded less than 1 and so on.

Lemma 1 (see [35]). Let the compound function $x \in$ $\mathscr{L}_{2}\left(\left[-\vartheta_{2},-\vartheta_{1}\right] \longrightarrow \mathbb{R}^{n}\right)$, symmetric matrix $\mathscr{R}>0$, and $\vartheta_{2}>\vartheta_{1}>0$. The Legendre polynomials considered over the interval $\left[-\vartheta_{2},-\vartheta_{1}\right]$ are defined by 


$$
\mathbb{L}_{k}(u)=(-1)^{k} \sum_{l=0}^{k} \mathscr{P}_{l}^{k}\left(\frac{u+\vartheta_{2}}{\vartheta_{2}-\vartheta_{1}}\right)^{l}, \quad \forall k \in \mathbb{N},
$$

with $\mathscr{P}_{l}^{k}=(-1)^{l}\left(\begin{array}{c}k \\ j\end{array}\right)\left(\begin{array}{c}k+j \\ j\end{array}\right)$, where $k=0,1, \ldots, N$.

Then, for all $N \in \mathbb{N}$, the integral inequality

$$
\int_{-\vartheta_{2}}^{-\vartheta_{1}} x^{T}(u) \mathscr{R} x(u) \mathrm{d} u \geq \frac{1}{\vartheta_{2}-\vartheta_{1}} \Theta^{T}(x)(\mathscr{R} \otimes \mathscr{F}) \Theta(x),
$$

holds with $\mathscr{F}=\operatorname{diag}\{1,3, \ldots, 2 N+1\}, \quad \Theta^{T}(x)=\left[\Theta_{0}^{T}(x) \ldots\right.$ $\left.\Theta_{k}^{T}(x) \cdots \Theta_{N}^{T}(x)\right]$, and $\Theta_{k}=\int_{-\vartheta_{2}}^{-\vartheta_{1}} \mathbb{L}_{k}(u) x(u) \mathrm{d} u$.

Lemma 2 (see [11]). Let $v(t) \in \mathbb{R}^{n}, \mathscr{P}=\mathscr{P}^{T} \in \mathbb{R}^{n \times n}$, and $\mathscr{H} \in \mathbb{R}^{m \times n}$ such that $\operatorname{rank}(\mathscr{H})=g<n$, then the following statements are equivalent:

(a) $\nu^{T}(t) \mathscr{P} v(t)<0$, for any $v(t) \neq 0, \mathscr{H} v(t)=0$ (b) $\mathscr{H}^{\perp T} \mathscr{P} \mathscr{H}^{\perp}<0$

(c) $\exists \mathscr{M} \in \mathbb{R}^{n \times m}$ such that $\mathscr{P}+H e(\mathscr{M} \mathscr{H})<0$

Lemma 3 (see [36]). For matrices $\mathscr{E}_{a}, \mathscr{G}(t)$, and $\mathscr{E}_{b}$ with compatible dimensions and $\mathscr{G}^{T}(t) \mathscr{G}(t) \leq I$, the inequality

$$
\mathscr{E}_{a} \mathscr{G}(t) \mathscr{E}_{b}+\mathscr{E}_{a}^{T} \mathscr{G}^{T}(t) \mathscr{E}_{b}^{T} \leq \beta \mathscr{E}_{a}^{T} \mathscr{E}_{a}+\beta^{-1} \mathscr{E}_{b}^{T} \mathscr{E}_{b},
$$

holds for any $\beta>0$.

\section{Main Results}

Before providing the main results, first, we construct the following LKF related to Legendre polynomials as

$$
V(t)=V_{0}(t)+V_{1}(t)+V_{2}(t),
$$

where

$$
\begin{aligned}
& V_{0}(t)=\zeta^{T}(t) P_{N} \zeta(t) \\
& V_{1}(t)=\int_{t-\tau_{1}}^{t} x^{T}(v)\left[S_{1}+\left(v-t+\tau_{1}\right) R_{1}\right] x(v) \mathrm{d} v, \\
& V_{2}(t)=\int_{t-\tau_{2}}^{t-\tau_{1}} x^{T}(v)\left[S_{2}+\left(v-t+\tau_{2}\right) R_{2}\right] x(v) \mathrm{d} v, \\
& \zeta^{T}(t)=\left[\begin{array}{llll}
x^{T}(t) & \Sigma^{T}(x) & \Gamma^{T}(x)
\end{array}\right]^{T}, \\
& \Sigma^{T}(x)=\left[\begin{array}{llllll}
\Sigma_{0}^{T}(x) & \cdots & \Sigma_{k_{1}}^{T}(x) & \cdots & \Sigma_{d_{1}}^{T}(x)
\end{array}\right], \\
& \Upsilon^{T}(x)=\left[\begin{array}{llllll}
\Upsilon_{0}^{T}(x) & \cdots & \Upsilon_{k_{2}}^{T}(x) & \cdots & \Upsilon_{d_{2}}^{T}(x)
\end{array}\right] \text {, } \\
& \Sigma_{k_{1}}(x)=\int_{-\tau_{1}}^{0} \mathbb{L}_{1, k_{1}}(v) x(v) \mathrm{d} v, \quad k_{1}=0,1, \ldots, d_{1}+1, \\
& \Upsilon_{k_{2}}(x)=\int_{-\tau_{2}}^{-\tau_{1}} \mathbb{L}_{2, k_{2}}(v) x(v) \mathrm{d} v, \quad k_{2}=0,1, \ldots, d_{2}+1 \text {. }
\end{aligned}
$$

Next, Theorem 1 gives the sufficient conditions for guaranteeing the closed-loop system (2) to be asymptotically stable in mean square sense. Based on Theorem 1 and Lemma 3, sufficient co-design conditions of controller gain and triggering matrix are derived via a set of LMIs in Theorem 2.

Theorem 1. For given scalars $d_{1}, d_{2}, \tau, \tau_{1}, \tau_{2}, \rho, \delta$, and $\bar{\alpha}$, under the proposed integral-based ETS (2) and controller gain $K$, the closed-loop system (2) is asymptotically stable in mean square sense if there exist symmetric matrices $P_{N}, \Phi>0$, $S_{1}>0, S_{2}>0, R_{1}>0$, and $R_{2}>0$ and matrices $U, X_{1}$, and $X_{2}$ such that

$$
\widehat{P}_{N}>0
$$

$$
\Pi+H e(\mathscr{W} \mathscr{V})<0
$$

where

$$
\begin{aligned}
\widehat{P}_{N}= & P_{N}+\operatorname{diag}\left\{0, \frac{\left(\mathscr{F}_{1} \otimes S_{1}\right)}{\tau_{1}}, \frac{\left(\mathscr{F}_{2} \otimes S_{2}\right)}{\tau}\right\}, \\
\Pi= & \operatorname{He}\left(H_{N}^{T} P_{N} M_{N}\right)+\delta \mathscr{I}_{1}^{T} \Phi \mathscr{I}_{1}-\rho \mathscr{I}_{2}^{T} \bar{F} \mathscr{I}_{2} \\
& +\operatorname{diag}\left\{0_{n}, \Pi_{12}, 0_{n}, \Pi_{14}, \Pi_{15}, \Pi_{16}, \Pi_{17}, \Pi_{18}\right\},
\end{aligned}
$$




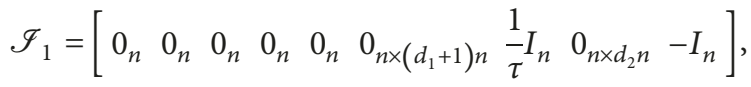

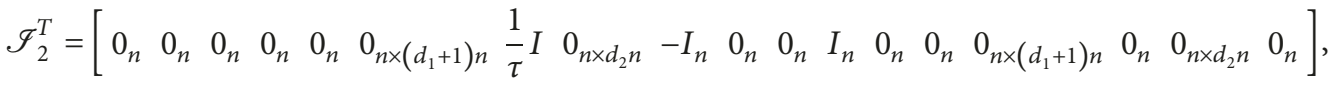

$$
\begin{aligned}
& \bar{F}=\left[\begin{array}{cc}
\bar{F}_{1} & \bar{F}_{2} \\
\bar{F}_{2}^{T} & I
\end{array}\right], \\
& \bar{F}_{1}=\frac{F_{1}^{T} F_{2}+F_{2}^{T} F_{1}}{2}, \\
& \bar{F}_{2}=\frac{F_{1}^{T}+F_{2}^{T}}{2}, \\
& \Pi_{12}=S_{1}+\tau_{1} R_{1}, \\
& \Pi_{14}=-S_{1}+S_{2}+\tau R_{2} \\
& \Pi_{15}=-S_{2}, \\
& \Pi_{16}=-\frac{\left(\mathscr{F}_{1} \otimes R_{1}\right)}{\tau_{1}}, \\
& \Pi_{17}=-\frac{\left(\mathscr{F}_{2} \otimes R_{2}\right)}{\tau}, \\
& \Pi_{18}=-\Phi, \\
& \mathscr{W}=\left[\begin{array}{llllllll}
W_{1}^{T} & W_{2}^{T} & 0_{n} & 0_{n} & 0_{n} & 0_{n \times\left(d_{1}+1\right) n} & 0_{n \times\left(d_{2}+1\right) n} & 0_{n}
\end{array}\right]^{T},
\end{aligned}
$$

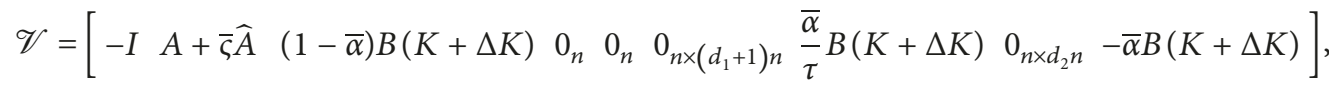

$$
\begin{aligned}
& M_{N}=\left[\begin{array}{cccccccc}
I_{n} & 0_{n} & 0_{n} & 0_{n} & 0_{n} & 0_{n \times\left(d_{1}+1\right) n} & 0_{n \times\left(d_{2}+1\right) n} & 0_{n} \\
0_{\left(d_{1}+1\right) n \times n} & \mathscr{L}_{d_{1}}(0) & 0_{\left(d_{1}+1\right) n \times n} & -\mathscr{L}_{d_{1}}\left(-\tau_{1}\right) & 0_{\left(d_{1}+1\right) n \times n} & \Lambda_{d_{1}} & 0_{\left(d_{1}+1\right) n \times\left(d_{2}+1\right) n} & 0_{\left(d_{1}+1\right) n \times n} \\
0_{\left(d_{2}+1\right) n \times n} & 0_{\left(d_{2}+1\right) n \times n} & 0_{\left(d_{2}+1\right) n \times n} & \mathscr{L}_{d_{2}}\left(-\tau_{1}\right) & -\mathscr{L}_{d_{2}}\left(-\tau_{2}\right) & 0_{\left(d_{2}+1\right) n \times\left(d_{1}+1\right) n} & \Lambda_{d_{2}} & 0 \\
0
\end{array}\right. \\
& H_{N}=\left[\begin{array}{cccccc}
0_{n} & I_{n} & 0_{n \times 3 n} & 0_{n \times\left(d_{1}+1\right) n} & 0_{n \times\left(d_{2}+1\right) n} & 0_{n} \\
0{ }_{\left(d_{1}+1\right) n \times n} & 0_{\left(d_{1}+1\right) n \times n} & 0_{\left(d_{1}+1\right) n \times 3 n} & I_{\left(d_{1}+1\right) n} & 0_{\left(d_{1}+1\right) n \times\left(d_{2}+1\right) n} & 0_{\left(d_{1}+1\right) n \times n} \\
0{ }_{\left(d_{2}+1\right) n \times n} & 0_{\left(d_{2}+1\right) n \times n} & 0_{\left(d_{2}+1\right) n \times 3 n} & 0_{\left(d_{1}+1\right) n \times\left(d_{2}+1\right) n} & \left.I d_{2}+1\right) n & 0_{\left(d_{2}+1\right) n \times n}
\end{array}\right], \\
& \mathscr{L}_{d_{1}}(0)=\left[\begin{array}{c}
I \\
I \\
\vdots \\
I
\end{array}\right] \\
& \mathscr{L}_{d_{1}}\left(-\tau_{1}\right)=\left[\begin{array}{c}
I \\
-I \\
\vdots \\
(-1)^{d_{1}} I
\end{array}\right]
\end{aligned}
$$




$$
\begin{aligned}
& \mathscr{L}_{d_{2}}\left(-\tau_{1}\right)=\left[\begin{array}{c}
I \\
I \\
\vdots \\
I
\end{array}\right] \\
& \mathscr{L}_{d_{2}}\left(-\tau_{2}\right)=\left[\begin{array}{c}
I \\
-I \\
\vdots \\
(-1)^{d_{2}} I
\end{array}\right] \\
& \Lambda_{d_{1}}=\left[\begin{array}{ccc}
\lambda_{d_{1} 0}^{0} I & \cdots & \lambda_{d_{1} 0}^{d_{1}} I \\
\vdots & \ddots & \vdots \\
\lambda_{d_{1} d_{1}}^{0} I & \cdots & \lambda_{d_{1} d_{1}}^{d_{1}} I
\end{array}\right] \\
& \lambda_{d_{1} k_{1}}^{i}= \begin{cases}\frac{-(2 i+1)\left(1-(-1)^{k_{1}+i}\right)}{\tau_{1}}, & i \leq k_{1}, \\
0, & i>k_{1},\end{cases} \\
& \Lambda_{d_{2}}=\left[\begin{array}{ccc}
\lambda_{d_{2} 0}^{0} I & \cdots & \lambda_{d_{2} 0}^{d_{2}} I \\
\vdots & \ddots & \vdots \\
\lambda_{d_{2} d_{2}}^{0} I & \cdots & \lambda_{d_{2} d_{2}}^{d_{2}} I
\end{array}\right], \\
& \lambda_{d_{2} k_{2}}^{i}= \begin{cases}\frac{-(2 i+1)\left(1-(-1)^{k_{2}+i}\right)}{\tau}, & i \leq k_{2}, \\
0, & i>k_{2} .\end{cases}
\end{aligned}
$$

Proof. Applying Lemma 1 to the constructed LKF (12), it gives that

$$
\begin{gathered}
\int_{t-\tau_{1}}^{t} x^{T}(v) S_{1} x(v) \mathrm{d} v \geq \frac{1}{\tau_{1}} \Omega^{T}(x)\left(\mathscr{F}_{1} \otimes S_{1}\right) \Omega(x), \\
\int_{t-\tau_{2}}^{t-\tau_{1}} x^{T}(v) S_{2} x(v) \mathrm{d} v \geq \frac{1}{\tau} \Gamma^{T}(x)\left(\mathscr{F}_{2} \otimes S_{2}\right) \Gamma(x) .
\end{gathered}
$$

According to (12), (17), and (18), it yields

$$
\begin{gathered}
V(t) \geq \zeta^{T}(t) \widehat{P}_{N} \zeta(t)+\int_{t-\tau_{1}}^{t} x^{T}(v)\left(v-t+\tau_{1}\right) R_{1} x(v) \mathrm{d} v \\
\cdot \int_{t-\tau_{2}}^{t-\tau_{1}} x^{T}(v)\left(v-t+\tau_{2}\right) R_{2} x(v) \mathrm{d} v .
\end{gathered}
$$

Now, we calculate the time derivative of $V(t)$ as

$$
\begin{aligned}
\dot{V}_{1}(t)= & 2 \zeta^{T}(t) P_{N} \dot{\zeta}(t)=2 \xi^{T}(t) H^{T} P_{N} M \xi(t) \\
\dot{V}_{2}(t)= & x^{T}(t)\left(S_{1}+\tau_{1} R_{1}\right) x(t)-x^{T}\left(t-\tau_{1}\right) S_{1} x\left(t-\tau_{1}\right) \\
& -\int_{t-\tau_{1}}^{t} x^{T}(v) R_{1} x(v) \mathrm{d} v \\
\dot{V}_{3}(t)= & x^{T}\left(t-\tau_{1}\right)\left(S_{2}+\tau_{2} R_{2}\right) x\left(t-\tau_{1}\right) \\
& -x^{T}\left(t-\tau_{2}\right) S_{2} x\left(t-\tau_{2}\right)-\int_{t-\tau_{2}}^{t-\tau_{1}} x^{T}(v) R_{2} x(v) \mathrm{d} v
\end{aligned}
$$

(19) where

From $R_{1}>0, R_{2}>0$, and $\widehat{P}_{N}>0$, the $\operatorname{LKF} V(t)$ is ensured to be positive.

$$
\xi^{T}(t)=\left[\begin{array}{llllllll}
\dot{x}^{T}(t) & x^{T}(t) & f^{T}\left(x\left(s_{k}\right)\right) & x^{T}\left(t-\tau_{1}\right) & x^{T}\left(t-\tau_{2}\right) & \Sigma^{T}(x) & Y^{T}(x) & \varepsilon^{T}(t)
\end{array}\right] .
$$


The derivatives of $\Sigma(x)$ and $\Upsilon(x)$ introduced in (12) are also computed as

$$
\begin{aligned}
& \dot{\Sigma}(x)=\left[\begin{array}{llllll}
\dot{\Sigma}_{0}^{T}(x) & \cdots & \dot{\Sigma}_{k_{1}}^{T}(x) & \cdots & \dot{\Sigma}_{d_{1}}^{T}(x)
\end{array}\right]^{T}, \\
& \dot{\Upsilon}(x)=\left[\begin{array}{llllll}
\dot{\Upsilon}_{0}^{T}(x) & \cdots & \dot{\Upsilon}_{k_{2}}^{T}(x) & \cdots & \dot{\Upsilon}_{d_{2}}^{T}(x)
\end{array}\right]^{T},
\end{aligned}
$$

where

$$
\begin{aligned}
\dot{\Sigma}_{k_{1}}(x)= & \mathbb{L}_{1, k_{1}}(0) x(t)-\mathbb{L}_{1, k_{1}}\left(-\tau_{1}\right) x\left(t-\tau_{1}\right) \\
& -\int_{-\tau_{1}}^{0} \dot{\mathbb{L}}_{1, k_{1}}(v) x(t+v) \mathrm{d} v, \\
\dot{\Upsilon}_{k_{2}}(x)= & \mathbb{L}_{2, k_{2}}\left(-\tau_{1}\right) x\left(t-\tau_{1}\right)-\mathbb{L}_{2, k_{2}}\left(-\tau_{2}\right) x\left(t-\tau_{2}\right) \\
& -\int_{-\tau_{2}}^{-\tau_{1}} \dot{\mathbb{L}_{2, k_{2}}}(v) x(t+v) \mathrm{d} v .
\end{aligned}
$$

Based on the Legendre polynomial properties presented in [35], one has

$$
\begin{aligned}
& \dot{\Sigma}_{k_{1}}(x)=x(t)-(-1)^{k_{1}} x\left(t-\tau_{1}\right)-\sum_{i=0}^{k_{1}-1} \lambda_{d_{1} k_{1}}^{i} \Sigma_{i}(x), \\
& \dot{\Upsilon}_{k_{2}}(x)=x\left(t-\tau_{1}\right)-(-1)^{k_{2}} x\left(t-\tau_{2}\right)-\sum_{i=0}^{k_{2}-1} \lambda_{d_{2} k_{2}}^{i} \Upsilon_{i}(x),
\end{aligned}
$$

which lead to

$$
\begin{aligned}
& \dot{\Sigma}(x)=\mathscr{L}_{d_{1}}(0) x(t)-\mathscr{L}_{d_{1}}\left(-\tau_{1}\right) x\left(t-\tau_{1}\right)+\Lambda_{d_{1}} \Sigma(x), \\
& \dot{\Upsilon}(x)=\mathscr{L}_{d_{2}}\left(-\tau_{1}\right) x(t)-\mathscr{L}_{d_{2}}\left(-\tau_{2}\right) x\left(t-\tau_{2}\right)+\Lambda_{d_{2}} \Upsilon(x) .
\end{aligned}
$$

To ensure the closed-loop system (2) to be asymptotically stable, one just needs

$$
\begin{aligned}
\dot{V}(t)= & 2 \xi^{T}(t) H^{T} P_{N} M \xi(t)+x^{T}(t)\left(S_{1}+\tau_{1} R_{1}\right) x(t) \\
& -x^{T}\left(t-\tau_{1}\right) S_{1} x\left(t-\tau_{1}\right)+x^{T}\left(t-\tau_{1}\right)\left(S_{2}+\tau R_{2}\right) x \\
& \cdot\left(t-\tau_{1}\right)-x^{T}\left(t-\tau_{2}\right) S_{2} x\left(t-\tau_{2}\right) \\
& -\int_{t-\tau_{1}}^{t} x^{T}(v) R_{1} x(v) \mathrm{d} v-\int_{t-\tau_{2}}^{t-\tau_{1}} x^{T}(v) R_{2} x(v) \mathrm{d} v<0 .
\end{aligned}
$$

Revisiting the triggering condition (5), one can get

$$
\varepsilon^{T}(t) \Phi \varepsilon(t)<\delta \xi^{T}(t) \mathscr{F}_{1}^{T} \Phi \mathscr{I}_{1} \xi(t) .
$$

The integral terms in (26) are handled by Lemma 1, which yield

$$
\begin{aligned}
& -\int_{t-\tau_{1}}^{t} x^{T}(v) R_{1} x(v) \mathrm{d} v \leq-\frac{1}{\tau_{1}} \Sigma^{T}(x)\left(\mathscr{F}_{1} \otimes R_{1}\right) \Sigma(x), \\
& -\int_{t-\tau_{2}}^{t-\tau_{1}} x^{T}(v) R_{2} x(v) \mathrm{d} v \leq-\frac{1}{\tau} \Upsilon^{T}(x)\left(\mathscr{F}_{2} \otimes R_{2}\right) \Upsilon(x) .
\end{aligned}
$$

Then, according to Assumption 1, one has

$$
\begin{gathered}
{\left[\begin{array}{c}
\hat{x}\left(s_{k}\right) \\
f\left(\widehat{x}\left(s_{k}\right)\right)
\end{array}\right]^{T}\left[\begin{array}{cc}
\bar{F}_{1} & \bar{F}_{2} \\
\bar{F}_{2}^{T} & I
\end{array}\right]\left[\begin{array}{c}
\hat{x}\left(s_{k}\right) \\
f\left(\widehat{x}\left(s_{k}\right)\right)
\end{array}\right]} \\
=\left[\begin{array}{c}
\int_{t-\tau_{2}}^{t-\tau_{1}} \frac{1}{\tau} x(v) \mathrm{d} v-\varepsilon(t) \\
f\left(\widehat{x}\left(s_{k}\right)\right)
\end{array}\right]^{T}\left[\begin{array}{ll}
\bar{F}_{1} & \bar{F}_{2} \\
\bar{F}_{2}^{T} & I
\end{array}\right] \\
\quad\left[\begin{array}{c}
\int_{t-\tau_{2}}^{t-\tau_{1}} \frac{1}{\tau} x(v) \mathrm{d} v-\varepsilon(t) \\
f\left(\hat{x}\left(s_{k}\right)\right)
\end{array}\right] \leq 0,
\end{gathered}
$$

which is rewritten as

$$
-\rho \xi^{T}(t) \mathscr{F}_{2}^{T} \bar{F}_{2} \xi(t) \geq 0,
$$

for any $\rho>0$.

Combining (27)-(29), (3) holds if the following inequality is satisfied:

$$
\xi^{T}(t) \Pi \xi(t)<0
$$

According to the description of system (2) and Lemma 2, we have

$$
\mathbb{E}\left\{\xi^{T}(t)(\Pi+\operatorname{He}(\overline{\mathscr{W}} \overline{\mathscr{V}})) \xi(t)\right\}<0,
$$

where

$$
\begin{aligned}
& \overline{\mathscr{W}}=\left[\begin{array}{lllllllll}
W_{1} & W_{2} & 0_{n} & 0_{n} & 0_{n} & 0_{n \times\left(d_{1}+1\right) n} & 0_{n \times\left(d_{2}+1\right) n} & 0_{n}
\end{array}\right]^{T}, \\
& \overline{\mathscr{V}}=\left[\begin{array}{llllllll}
-I & A+\Delta A(t) & \left(1-\alpha\left(s_{k}\right)\right) B(K+\Delta K) & 0_{n} & 0_{n} & 0_{n \times\left(d_{1}+1\right) n} \frac{\alpha\left(s_{k}\right)}{\tau} B(K+\Delta K) & 0_{n \times d_{2} n}-\alpha\left(s_{k}\right) B(K+\Delta K)
\end{array}\right] .
\end{aligned}
$$

Noting that

$$
\mathbb{E}\left\{\xi^{T}(t) \mathrm{He}(\overline{\mathscr{W}} \overline{\mathscr{V}}) \xi(t)\right\}=\xi^{T}(t) \mathrm{He}(\mathscr{W} \mathscr{V}) \xi(t)=0,
$$

it is clear that (33) is equivalent to (15).

Remark 6. In the existing result [31] concerning integralbased event-triggered control issue, an approximation method is adopted to handle the integral term induced by the triggering condition. Based on this method, approximation error is an inevitable factor, which could introduce conservativeness in controller design. Nevertheless, with the help of Legendre polynomials and their properties, such approximation error is removed in this paper, and less conservative results could be obtained. 
Theorem 2. For given scalars $d_{1}, d_{2}, \tau, \tau_{1}, \tau_{2}, \rho, \delta$, and $\bar{\alpha}$, under the proposed integral-based ETS (2), the closed-loop system (2) is asymptotically stable in mean square sense if there exist symmetric matrices $P_{N}, \Phi>0, S_{1}>0, S_{2}>0$, $R_{1}>0$, and $R_{2}>0$ and matrices $U, W_{1}, W_{2}, Z$, and $N$ such that

$$
\left[\begin{array}{ccc}
\widehat{\Pi} & \beta \mathscr{W} B \mathscr{E}_{a} & \mathscr{E}_{b}^{T} \\
* & -\beta I & 0 \\
* & * & -\beta I
\end{array}\right]<0,
$$

$$
\begin{aligned}
& \widehat{\Pi}=\left[\begin{array}{cc}
\Pi+H e(\tilde{\mathscr{W}} \tilde{\mathscr{V}}) & \Pi_{2}^{T} \\
* & -\mu_{3} H e(N)
\end{array}\right] \\
& \mathscr{E}_{a}=E_{a},
\end{aligned}
$$

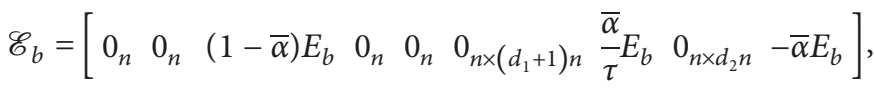

$$
\begin{aligned}
& \Pi_{2}=\left[\begin{array}{llllllllll}
\Pi_{21} & \Pi_{22} & \Pi_{23} & 0_{n} & 0_{n} & 0_{n} & 0_{n \times\left(d_{1}+1\right) n} & \Pi_{24} & 0_{n \times d_{2} n} & \Pi_{25}
\end{array}\right], \\
& \Pi_{21}=\left(\mu_{1} B N-W_{1} B\right)^{T} \text {, } \\
& \Pi_{22}=\left(\mu_{2} B N-W_{2} B\right)^{T}, \\
& \Pi_{23}=(1-\bar{\alpha}) \mu_{3} Z \text {, } \\
& \Pi_{24}=\frac{\bar{\alpha}}{\tau} \mu_{3} Z \\
& \Pi_{25}=-\bar{\alpha} \mu_{3} Z \text {, } \\
& \widetilde{\mathscr{W}}=\left[\begin{array}{ccccccccc}
I & 0_{n} & 0_{n} & 0_{n} & 0_{n} & 0_{n \times\left(d_{1}+1\right) n} & 0_{n \times\left(d_{2}+1\right) n} & 0_{n} & 0_{n} \\
0_{n} & I & 0_{n} & 0_{n} & 0_{n} & 0_{n \times\left(d_{1}+1\right) n} & 0_{n \times\left(d_{2}+1\right) n} & 0_{n} & 0_{n}
\end{array}\right]^{T}, \\
& \tilde{\mathscr{V}}=\left[\begin{array}{lllllllll}
-W_{1} & W_{1}(A+\bar{\zeta} \widehat{A}) & (1-\bar{\alpha}) \mu_{1} B Z & 0_{n} & 0_{n} & 0_{n \times\left(d_{1}+1\right) n} & \frac{\bar{\alpha}}{\tau} \mu_{1} B Z & 0_{n \times d_{2} n}-\bar{\alpha} \mu_{1} B Z & 0_{n} \\
-W_{2} & W_{2}(A+\bar{\varsigma} \widehat{A}) & (1-\bar{\alpha}) \mu_{2} B Z & 0_{n} & 0_{n} & 0_{n \times\left(d_{1}+1\right) n} & \frac{\bar{\alpha}}{\tau} \mu_{2} B Z & 0_{n \times d_{2} n}-\bar{\alpha} \mu_{2} B Z & 0_{n}
\end{array}\right]
\end{aligned}
$$

Moreover, the controller gain is obtained as $K=N^{-1} Z$.

$$
\Pi+H e(\mathscr{W} \hat{\mathscr{V}})+H e(\mathscr{W} \Delta \mathscr{V})<0,
$$

Proof. It is noted that (15) can be written as where

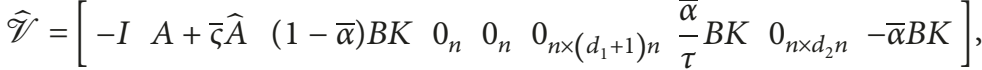

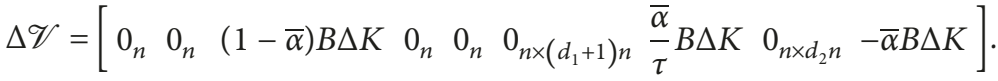

Based on Lemma 3, one has

$$
\begin{aligned}
H e(\mathscr{W} \Delta \mathscr{V})= & H e\left(\mathscr{W} B \mathscr{E}_{a} G(t) \mathscr{E}_{b}\right) \leq \beta \mathscr{W} B \mathscr{E}_{a}\left(\mathscr{W} B \mathscr{E}_{a}\right)^{T} \\
& +\beta^{-1} \mathscr{E}_{b}^{T} \mathscr{E}_{b} .
\end{aligned}
$$

With the help of Lemma 2, (15) is ensured by

$$
\mathscr{H}^{\perp^{T}}\left[\begin{array}{cc}
\Pi+H e(\mathscr{W} \hat{\mathscr{V}})+\beta \mathscr{W} B \mathscr{E}_{a}\left(\mathscr{W} B \mathscr{E}_{a}\right)^{T}+\beta^{-1} \mathscr{E}_{b}^{T} \mathscr{E}_{b} & 0 \\
0 & 0
\end{array}\right] \mathscr{H}^{\perp}<0,
$$


where

$$
\begin{aligned}
& \mathscr{H}^{\perp}=\left[\begin{array}{c}
\mathscr{H}_{1}^{\perp} \\
\mathscr{H}_{2}^{\perp}
\end{array}\right], \\
& \mathscr{H}_{1}^{\perp}=\operatorname{diag}\{I, I, I, I, I, \underbrace{I, \ldots, I}_{d_{1}+d_{2}+2}, I\},
\end{aligned}
$$

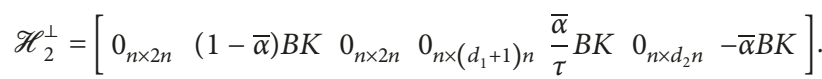

By applying Lemma 2 to (41), it leads to

$$
\begin{aligned}
& {\left[\begin{array}{cc}
\Pi+H e(\mathscr{W} \hat{\mathscr{V}})+\beta \mathscr{W} B \mathscr{E}_{a}\left(\mathscr{W} B \mathscr{E}_{a}\right)^{T}+\beta^{-1} \mathscr{E}_{b}^{T} \mathscr{E}_{b} & 0 \\
0 & 0
\end{array}\right]} \\
& \quad+\operatorname{He}(\mathscr{M} \mathscr{H})<0,
\end{aligned}
$$

where

$$
\begin{aligned}
& \mathscr{M}=\left[\begin{array}{lllllll}
\mathscr{M}_{1} & \mathscr{M}_{2} & 0_{m \times 3 n} & 0_{m \times\left(d_{1}+1\right) n} & 0_{m \times\left(d_{2}+1\right) n} & 0_{m \times n} & \mu_{3} N^{T}
\end{array}\right]^{T},
\end{aligned}
$$

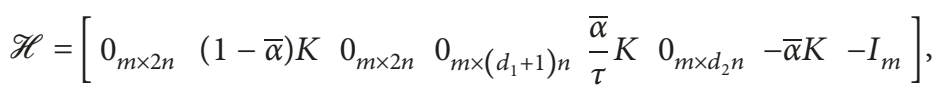

$$
\begin{aligned}
& \mathscr{M}_{1}=\left(\mu_{1} B N-W_{1} B\right)^{T} \\
& \mathscr{M}_{2}=\left(\mu_{2} B N-W_{2} B\right)^{T} .
\end{aligned}
$$

Using the Schur complement, it results in

$$
\left[\begin{array}{ccc}
\widehat{\Pi} & \beta \mathscr{W} B \mathscr{E}_{a} & \mathscr{E}_{b}^{T} \\
* & -\beta I & 0 \\
* & * & -\beta I
\end{array}\right]<0,
$$

where

$$
\widehat{\Pi}=\left[\begin{array}{cc}
\Pi+H e(\mathscr{W} \hat{\mathscr{V}}) & 0 \\
0 & 0
\end{array}\right]+H e(\mathscr{M} \mathscr{H})
$$

Therefore, (36) is ensured by (45).

Remark 7. To deal with the cyber-attacks satisfying Assumption 1, the well-known S-procedure is also used in [27], which will cause the difficulty of controller design by the conventional pre- and post-multiplying technique. In [27], the control matrix $B$ is assumed to be full column rank to solve this problem. However, a decomposition technique on the basis of Lemma 3 is adopted in Theorem 2, which removes such rank constraint.

Remark 8. The similar way in [10] can be utilized to derive the positive lower bound of inner-event time, which implies that the infinite triggering events over finite time interval are excluded via the proposed integral-based ETS (2).
When cyber-attacks are not considered, the closed-loop system (2) is rewritten as

$$
\begin{array}{r}
\dot{x}(t)=(A+\Delta A(t)) x(t)+B(K+\Delta K)\left(\frac{1}{\tau} \int_{t-\tau_{2}}^{t-\tau_{1}} x(v) \mathrm{d} v-\varepsilon(t)\right), \\
t \in\left[t_{k}, t_{k+1}\right) .
\end{array}
$$

Following the similar way to derive Theorem 2, the terms related with $f\left(x\left(s_{k}\right)\right)$ are removed, and the control synthesis conditions are produced in Corollary 1.

Corollary 1. For given scalars $d_{1}, d_{2}, \tau, \tau_{1}, \tau_{2}, \rho$, and $\delta$, considering the event-triggered scheme (2), the closed-loop system (3) is asymptotically stable if there exist symmetric matrices $P_{N}, \Phi>0, S_{1}>0, S_{2}>0, R_{1}>0$, and $R_{2}>0$ and matrices $W_{1}, W_{2}, Z$, and $N$ such that

$$
\left[\begin{array}{ccc} 
& \widehat{P}_{N}>0, \\
* & \beta \widehat{\mathscr{W}} B \widehat{\mathrm{E}}_{a} & \widehat{\mathrm{E}}_{b}^{T} \\
* & * & 0 \\
* I & -\beta I
\end{array}\right]<0,
$$

where 


$$
\begin{aligned}
& \widehat{\Xi}=\left[\begin{array}{cc}
\Xi & \Xi_{2} \\
\Xi_{2}^{T} & -\mu_{3} H e(W)
\end{array}\right]+H e(\breve{\mathscr{W}} \breve{\mathscr{V}}), \\
& \widehat{\mathscr{E}}_{a}=E_{a} \\
& \widehat{\mathscr{W}}=\left[\begin{array}{lllllll}
W_{1}^{T} & W_{2}^{T} & 0_{n} & 0_{n} & 0_{n \times\left(d_{1}+1\right) n} & 0_{n \times\left(d_{2}+1\right) n} & 0_{n}
\end{array}\right]^{T}, \\
& \widehat{\mathscr{E}}_{b}=\left[\begin{array}{llll}
0_{n \times 4 n} & 0_{n \times\left(d_{1}+1\right) n} \frac{1}{\tau} E_{b} & 0_{n \times d_{2} n} & -E_{b}
\end{array}\right], \\
& \Xi=H e\left(\widehat{H}_{N}^{T} P_{N} \widehat{M}_{N}\right)+\delta \mathscr{I}_{4}^{T} \Phi \mathscr{I}_{4}+\operatorname{diag}\left\{0_{n}, \Xi_{12}, \Xi_{13}, \Xi_{14}, \Xi_{15}, \Xi_{16}, \Xi_{17}\right\}, \\
& \mathscr{I}_{4}=\left[\begin{array}{llll}
0_{n \times 4 n} & 0_{n \times\left(d_{1}+1\right) n} \frac{1}{\tau} I & 0_{n \times d_{2} n}-I
\end{array}\right], \\
& \Xi_{12}=S_{1}+\tau_{1} R_{1} \\
& \Xi_{13}=-S_{1}+S_{2}+\tau R_{2} \\
& \Xi_{14}=-S_{2} \\
& \Xi_{15}=-\frac{\left(\mathscr{F}_{1} \otimes R_{1}\right)}{\tau_{1}}, \\
& \Xi_{16}=-\frac{\left(\mathscr{F}_{2} \otimes R_{2}\right)}{\tau}, \\
& \Xi_{17}=-\Phi \\
& \Xi_{2}^{T}=\left[\begin{array}{llllll}
\Xi_{21} & \Xi_{22} & 0_{n} & 0_{n} & 0_{n \times\left(d_{1}+1\right) n} \frac{\mu_{3}}{\tau} Z & 0_{n \times d_{2} n}-\mu_{3} Z
\end{array}\right], \\
& \Xi_{21}=\left(\mu_{1} B N-W_{1} B\right)^{T} \\
& \Xi_{22}=\left(\mu_{2} B N-W_{2} B\right)^{T} \\
& \breve{\mathscr{W}}=\left[\begin{array}{ccccccc}
I & 0_{n} & 0_{n \times 2 n} & 0_{n \times\left(d_{1}+1\right) n} & 0_{n \times\left(d_{2}+1\right) n} & 0_{n} & 0_{n} \\
0_{n} & I & 0_{n \times 2 n} & 0_{n \times\left(d_{1}+1\right) n} & 0_{n \times\left(d_{2}+1\right) n} & 0_{n} & 0_{n}
\end{array}\right]^{T}, \\
& \breve{\mathscr{V}}=\left[\begin{array}{cccccccc}
-W_{1} & W_{1}(A+\bar{\zeta} \widehat{A}) & 0_{n \times 2 n} & 0_{n \times\left(d_{1}+1\right) n} & \frac{\mu_{1}}{\tau} B Z & 0_{n \times d_{2} n} & -\mu_{1} B Z & 0 \\
-W_{2} & W_{2}(A+\bar{\zeta} \widehat{A}) & 0_{n \times 2 n} & 0_{n \times\left(d_{1}+1\right) n} \frac{\mu_{2}}{\tau} B Z & 0_{n \times d_{2} n} & -\mu_{2} B Z & 0
\end{array}\right] .
\end{aligned}
$$




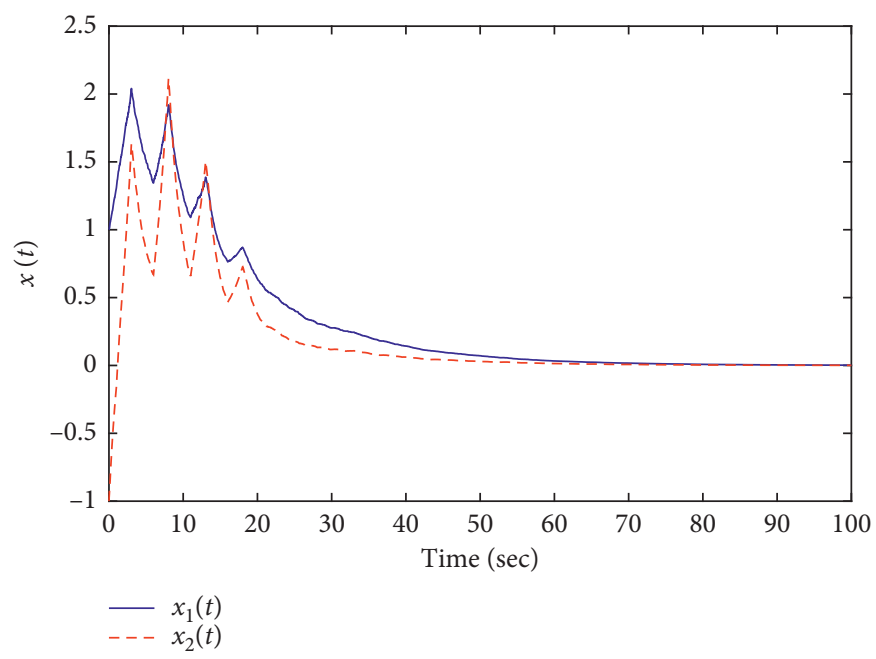

Figure 2: The system state responses under the controller solved by Theorem 2 .

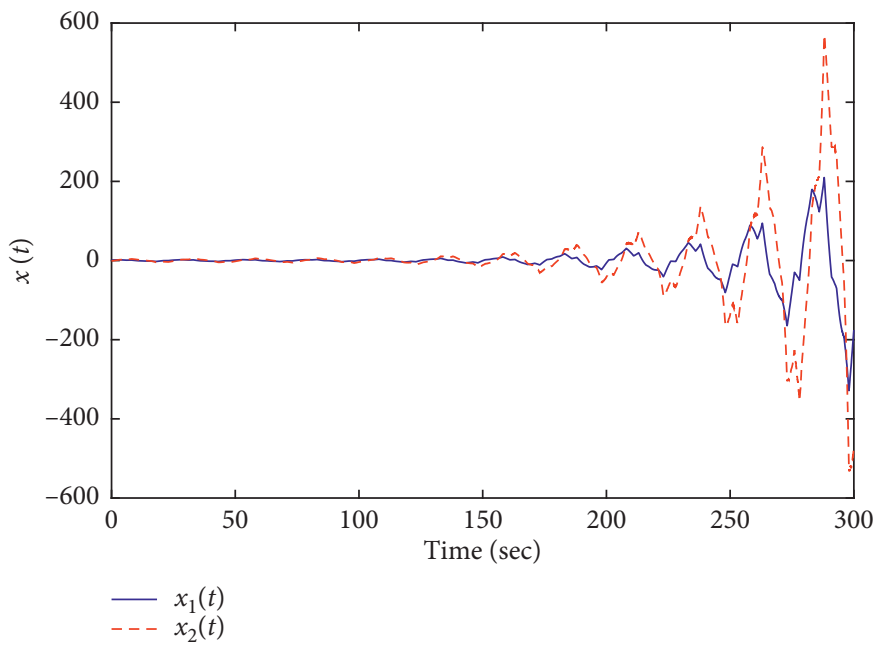

Figure 3: The system state responses under the controller solved by Corollary 1 .

\section{Numerical Example}

Example 1. The parameters of system (1) and cyber-attack function are given as

$$
\begin{aligned}
A & =\left[\begin{array}{cc}
0.1 & 0 \\
0.8 & -0.2
\end{array}\right], \\
B & =\left[\begin{array}{c}
0.5 \\
1
\end{array}\right], \\
\widehat{A} & =\left[\begin{array}{cc}
0.3 & 0 \\
0 & 0.2
\end{array}\right], \\
\bar{\zeta} & =0.8, \\
E_{a} & =0.02, \\
E_{b} & =\left[\begin{array}{ll}
0.2 & 0.1
\end{array}\right] .
\end{aligned}
$$

Assume the cyber-attack function as

$$
f\left(x\left(s_{k}\right)\right)=\operatorname{sat}\left(x\left(s_{k}\right)\right)= \begin{cases}\bar{x}_{i}, & x_{i}\left(s_{k}\right)>\bar{x}_{i}, \\ x_{i}\left(s_{k}\right), & -\bar{x}_{i} \leq x_{i}\left(s_{k}\right) \leq \bar{x}_{i}, i=1,2, \\ -\bar{x}_{i}, & x_{i}\left(s_{k}\right)<-\bar{x}_{i},\end{cases}
$$

where the saturation levels are $\bar{x}_{1}=0.5$ and $\bar{x}_{2}=0.4$. The cyber-attack function satisfies Assumption 1 with $F_{1}=\operatorname{diag}\{0,0\}$ and $F_{2}=\operatorname{diag}\{1.2,1.2\}$.

By choosing $\bar{\alpha}=0.8, d_{1}=d_{2}=1, \tau=0.1, \tau_{1}=0.04$, $\tau_{2}=0.14, \rho=15, \delta=0.01, \mu_{1}=\mu_{2}=1$, and $\mu_{3}=0.1$, the controller gain $K$ and triggering parameter $\Phi$ solved by Theorem 2 are given as

$$
\begin{aligned}
& K=\left[\begin{array}{ll}
-0.4919 & -0.6554
\end{array}\right], \\
& \Phi=\left[\begin{array}{ll}
284.6063 & -15.8585 \\
-15.8585 & 270.7435
\end{array}\right] .
\end{aligned}
$$

Then, by solving Corollary 1 where the cyber-attacks are not taken into account, it gives the corresponding parameters $K$ and $\Phi$ as 


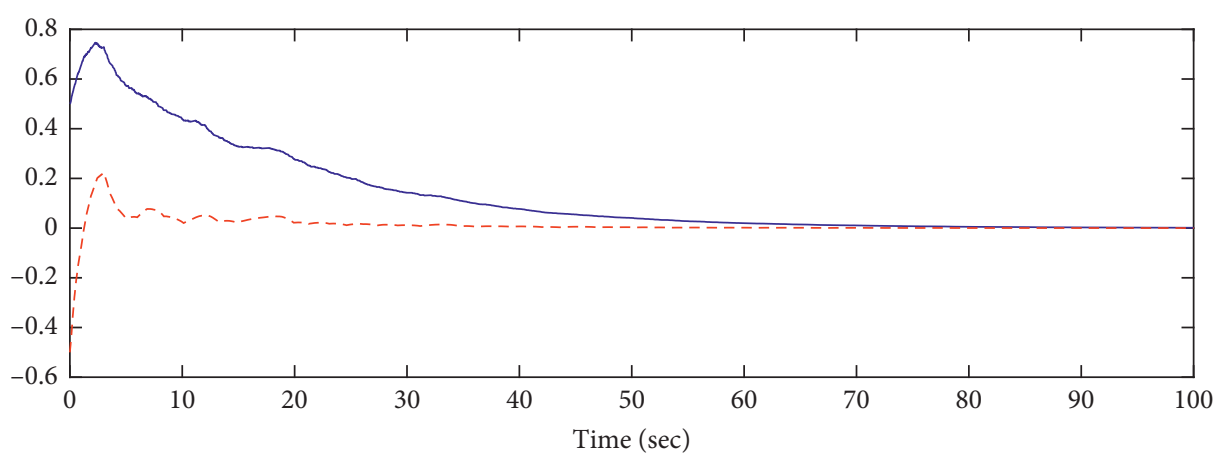

- $x_{1}(t)$ under the proposed integral-based ETS

- - $x_{2}(t)$ under the proposed integral-based ETS

(a)

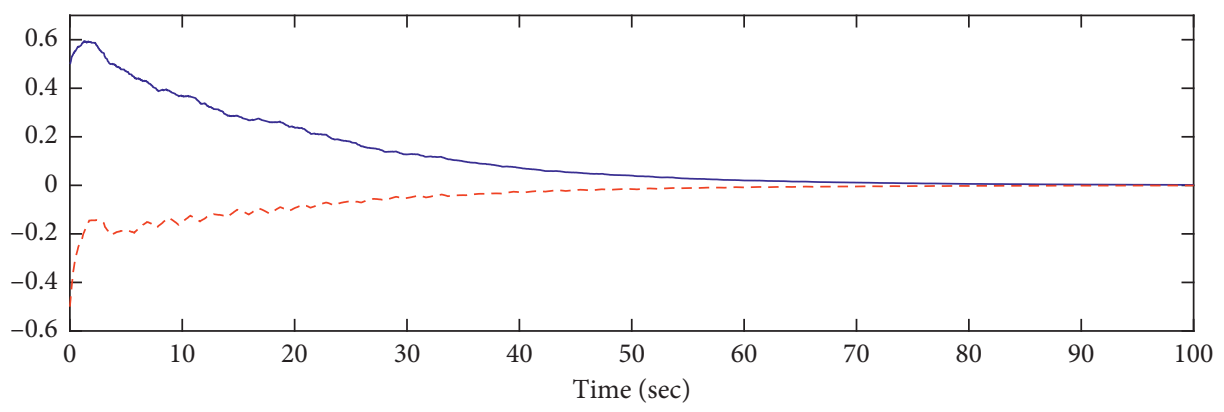

- $x_{1}(t)$ under the normal ETS

- - $x_{2}(t)$ under the normal ETS

(b)

FIGURE 4: The comparison of system state $x(t)$.

$$
\begin{aligned}
& K=\left[\begin{array}{ll}
-0.3050 & -0.3878
\end{array}\right], \\
& \Phi=\left[\begin{array}{cc}
127.6124 & -2.9095 \\
-2.9095 & 125.5567
\end{array}\right] .
\end{aligned}
$$

In the simulation, we choose the initial condition as $x(0)=\left[\begin{array}{ll}1 & -1\end{array}\right]^{T}$ and $G(t)=\sin (t)$. The system state responses under the controller solved by Theorem 2 and Corollary 1 are drawn in Figures 2 and 3, respectively.

From Figure 3, one can see that the cyber-attacks can cause system instability. When the cyber-attacks are considered in system analysis, the system state responses given in Figure 2 are stabilized by the designed controller solved by Theorem 2 , which also shows the effectiveness of the proposed method.

Moreover, to illustrate the effectiveness of the proposed integral-based ETS, the following simulations are implemented. For $\bar{\alpha}=0.7$ and the same parameters with above, the corresponding controller gain $K$ and triggering parameter $\Phi$ solved by Theorem 2 are given as

$$
\begin{aligned}
K & =\left[\begin{array}{ll}
-0.6818 & -1.0267
\end{array}\right], \\
\Phi & =\left[\begin{array}{ll}
307.9407 & -31.9729 \\
-31.9729 & 270.2628
\end{array}\right] .
\end{aligned}
$$

In the simulation, initial condition is chosen as $x(0)=\left[\begin{array}{ll}0.5 & -0.5\end{array}\right]^{T}$. For $d_{1}=d_{2}=1$, with the above parameters, the compared trajectories of system state under the

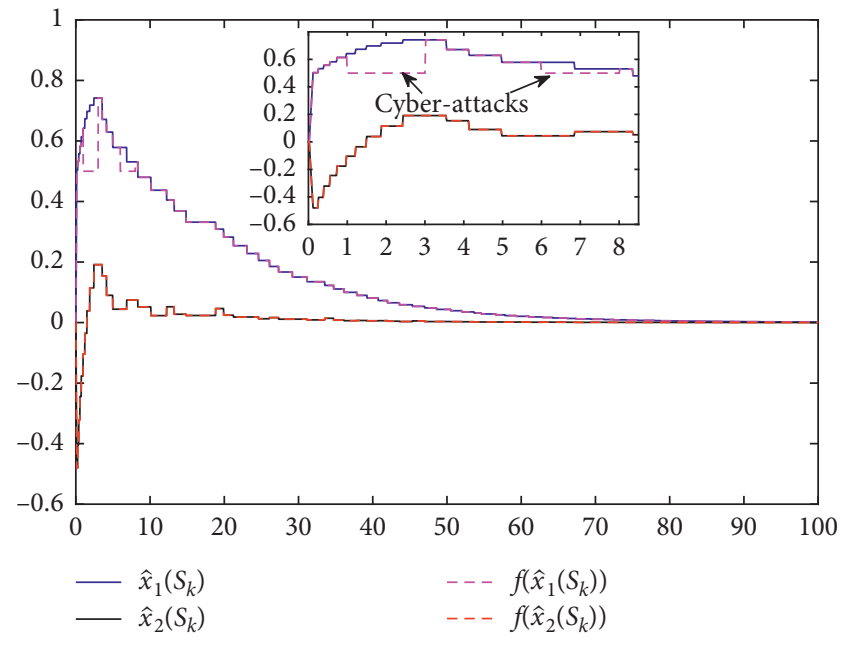

Figure 5: $x\left(s_{k}\right)$ and $f\left(x\left(s_{k}\right)\right)$ under the integral-based ETS (2).

proposed integral-based ETS (2) and the normal ETS (3) are depicted in Figure 4. The triggered signal $x\left(s_{k}\right)$ and attacked signal $f\left(x\left(s_{k}\right)\right)$ under these two ETSs are given in Figures 5 and 6 , respectively. Figure 7 illustrates the comparison of release time intervals.

From Figure 4, it is seen that the similar convergence times are performed under these two different ETSs. However, from Figure 7 and Table 1, the proposed integral- 


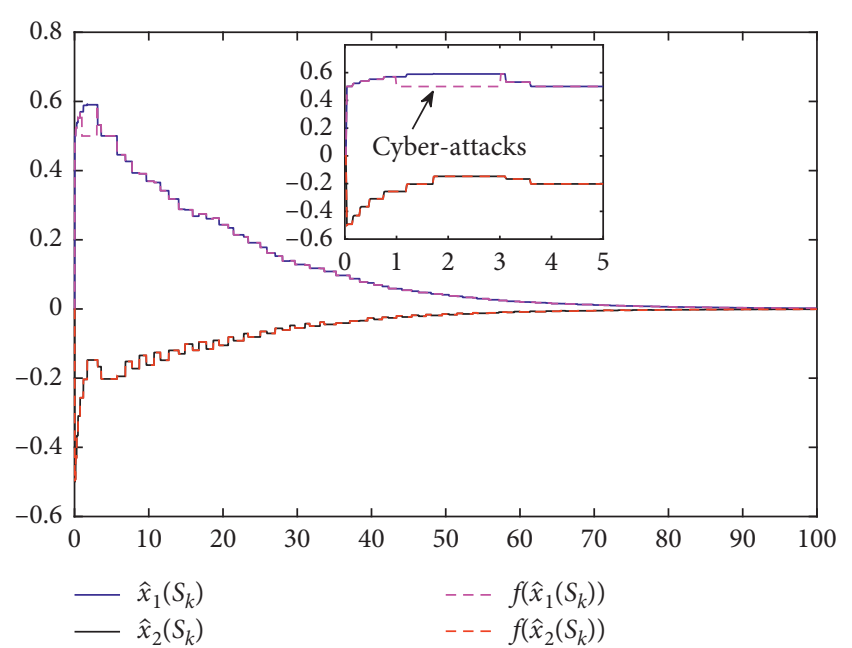

Figure 6: $x\left(s_{k}\right)$ and $f\left(x\left(s_{k}\right)\right)$ under the normal ETS (3).

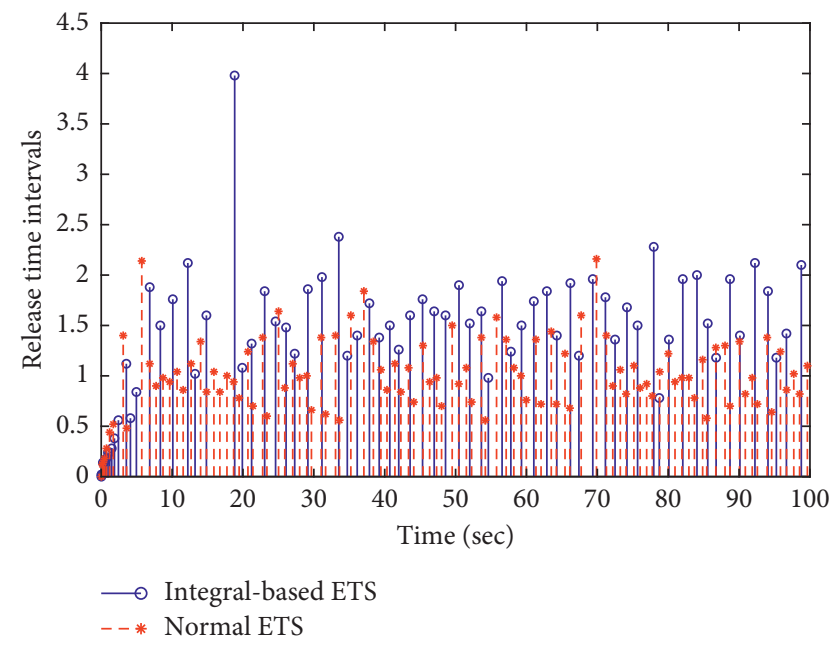

FIgURE 7: The comparison of release time intervals.

TABLE 1: Average triggering time intervals, $\bar{T}(s)$.

\begin{tabular}{lcc}
\hline & Integral-based ETS & Normal ETS \\
\hline $\bar{T}(s)$ & 1.37 & 1.01 \\
\hline
\end{tabular}

based ETS increases $26.26 \%$ of the average triggering time interval compared with the normal ETS. Consequently, these results show that the proposed integral-based ETS generates fewer trigger events and saves more network resources than the normal ETS.

\section{Conclusions}

This paper focuses on the study of the nonfragile integralbased event-triggered control issue for linear CPSs with transmission delay, gain variations, and cyber-attacks. An integral-based ETS is proposed to decrease the unnecessary data transmissions, which utilizes the mean of system state instead of the instant system information. A stochastic variable is used to model the randomly launched cyberattacks in the communication channel. To deal with the integral term induced by integral-based ETS, Legendre polynomials are introduced to construct a novel LKF. With the help of the LMI technique, some sufficient conditions are obtained to ensure the mean square stability of the closedloop system. Finally, the validity of the presented results is shown through a numerical example. Since the controllers are usually distributed at different nodes in real CPSs, the same triggering conditions and the same cyber-attacks for all communication channels maybe conservative. Thus, how to apply our proposed event-triggered scheme to the issue of decentralized security control against hybrid attacks is one of our future investigations. Furthermore, extending the proposed integral-based ETS to the finite time control $[37,38]$ and the chance-constrained control [39] will be another interesting research topic.

\section{Data Availability}

The data used to support the findings of this study are available from the corresponding author upon request.

\section{Conflicts of Interest}

All authors declare that they have no conflicts of interest.

\section{Acknowledgments}

This work was supported by the scholarship from China Scholarship Council under grant no. 201708320364, the National Natural Science Foundation of China under grant nos. 61773200 and 51877060, and the Key Project Foundation of Natural Science Research in Universities of Anhui Province in China (no. KJ2019A0645).

\section{References}

[1] P. Antsaklis, "Goals and challenges in cyber-physical systems research editorial of the editor in chief," IEEE Transactions on Automatic Control, vol. 59, no. 12, pp. 3117-3119, 2014.

[2] F. Harirchi and N. Ozay, "Guaranteed model-based fault detection in cyber-physical systems: a model invalidation approach," Automatica, vol. 93, pp. 476-488, 2018.

[3] T. Wang, X. Wei, T. Huang et al., "Cascading failures analysis considering extreme virus propagation of cyber-physical systems in smart grids," Complexity, vol. 2019, Article ID 7428458, 15 pages, 2019.

[4] C. Kwon and I. Hwang, "Reachability analysis for safety assurance of cyber-physical systems against cyber attacks," IEEE Transactions on Automatic Control, vol. 63, no. 7, pp. 22722279, 2018.

[5] Z. Cao, Y. Niu, and Y. Zou, "Adaptive neural sliding mode control for singular semi-Markovian jump systems against actuator attacks," IEEE Transactions on Systems, Man, and Cybernetics: Systems, pp. 1-11, 2019.

[6] Y. Mo, T. H.-J. Kim, K. Brancik et al., "Cyber-physical security of a smart grid infrastructure," Proceedings of the IEEE, vol. 100, no. 1, pp. 195-209, 2012.

[7] R. Kozik, M. Pawlicki, M. Choraś, and W. Pedrycz, "Practical employment of granular computing to complex application 
layer cyberattack detection," Complexity, vol. 2019, Article ID 5826737, 9 pages, 2019.

[8] D. Ding, Z. Wang, D. W. C. Ho, and G. Wei, "Distributed recursive filtering for stochastic systems under uniform quantizations and deception attacks through sensor networks," Automatica, vol. 78, pp. 231-240, 2017.

[9] X. Zhao, C. Liu, and E. Tian, "Finite-horizon tracking control for a class of stochastic systems subject to input constraints and hybrid cyber attacks," ISA Transactions, 2019.

[10] P. Tabuada, "Event-triggered real-time scheduling of stabilizing control tasks," IEEE Transactions on Automatic Control, vol. 52, no. 9, pp. 1680-1685, 2007.

[11] M. Shen, S. K. Nguang, and C. K. Ahn, "Quantized $H_{\infty}$ output control of linear Markov jump systems in finite frequency domain," IEEE Transactions on Systems, Man, and Cybernetics: Systems, vol. 49, no. 9, pp. 1901-1911, 2019.

[12] X. Xie, D. Yue, and C. Peng, "Relaxed real-time scheduling stabilization of discrete-time takagi-sugeno fuzzy systems via an alterable-weights-based ranking switching mechanism," IEEE Transactions on Fuzzy Systems, vol. 26, no. 6, pp. 3808-3819, 2018.

[13] X. Xie, Q. Zhou, D. Yue, and H. Li, "Relaxed control design of discrete-time Takagi-Sugeno fuzzy systems: an event-triggered real-time scheduling approach," IEEE Transactions on Systems, Man, and Cybernetics: Systems, vol. 48, no. 12, pp. 2251-2262, 2018.

[14] D. Yue, E. Tian, and Q.-L. Han, "A delay system method for designing event-triggered controllers of networked control systems," IEEE Transactions on Automatic Control, vol. 58, no. 2, pp. 475-481, 2013.

[15] S. Hu, D. Yue, Q. L. Han, X. Xie, X. Chen, and C. Dou, "Observer-based event-triggered control for networked linear systems subject to denial-of-Service attacks," IEEE Transactions on Cybernetics, pp. 1-13, 2019.

[16] S. Hu, D. Yue, X. Chen, Z. Cheng, and X. Xie, "Resilient $H_{\infty}$ filtering for event-triggered networked systems under nonperiodic DoS jamming attacks," IEEE Transactions on Systems, Man, and Cybernetics: Systems, pp. 1-12, 2019.

[17] Y. Liu and X. Hou, "Event-triggered consensus control for leader-following multiagent systems using output feedback," Complexity, vol. 2018, Article ID 6342683, 9 pages, 2018.

[18] E. Tian, Z. Wang, L. Zou, and D. Yue, "Probabilistic-constrained filtering for a class of nonlinear systems with improved static event-triggered communication," International Journal of Robust and Nonlinear Control, vol. 29, no. 5, pp. 1484-1498, 2019.

[19] Q. Lü and H. Li, "Event-triggered discrete-time distributed consensus optimization over time-varying graphs," Complexity, vol. 2017, Article ID 5385708, 12 pages, 2017.

[20] Z. Gu, T. Zhang, F. Yang, H. Zhao, and M. Shen, "A novel event-triggered mechanism for networked cascade control system with stochastic nonlinearities and actuator failures," Journal of the Franklin Institute, vol. 356, no. 4, pp. 1955-1974, 2019.

[21] S. Yan, M. Shen, S. K. Nguang, G. Zhang, and L. Zhang, "A distributed delay method for event-triggered control of T-S fuzzy networked systems with transmission delay," IEEE Transactions on Fuzzy Systems, vol. 27, no. 10, pp. 1963-1973, 2019.

[22] Z. Gu, P. Shi, D. Yue, and Z. Ding, "Decentralized adaptive event-triggered $H_{\infty}$ filtering for a class of networked nonlinear interconnected systems," IEEE Transactions on Cybernetics, vol. 99, no. 5, pp. 1-10, 2018.
[23] S. Yan, M. Shen, G. Zhang, and S. K. Nguang, "Reliable Ho output control of nonlinear systems with dynamic eventtriggered scheme," Journal of the Franklin Institute, vol. 356, no. 1, pp. 58-79, 2019.

[24] C. Peng, J. Li, and M. Fei, "Resilient event-triggering $H_{\infty}$ load frequency control for multi-area power systems with energylimited DoS attacks," IEEE Transactions on Power Systems, vol. 32, no. 5, pp. 4110-4118, 2017.

[25] S. Hu, D. Yue, X. Xie, X. Chen, and X. Yin, "Resilient eventtriggered controller synthesis of networked control systems under periodic dos jamming attacks," IEEE Transactions on Cybernetics, vol. 49, no. 12, pp. 4271-4281, 2019.

[26] D. Ding, Z. Wang, D. W. C. Ho, and G. Wei, "Observer-based event-triggering consensus control for multiagent systems with lossy sensors and cyber-attacks," IEEE Transactions on Cybernetics, vol. 47, no. 8, pp. 1936-1947, 2017.

[27] L. Zha, E. Tian, X. Xie, Z. Gu, and J. Cao, "Decentralized event-triggered $H_{\infty}$ control for neural networks subject to cyber-attacks," Information Sciences, vol. 457-458, pp. 141-155, 2018.

[28] J. Liu, E. Tian, X. Xie, and H. Lin, "Distributed event-triggered control for networked control systems with stochastic cyberattacks," Journal of the Franklin Institute, 2018.

[29] X. Zhou, Z. Gu, and F. Yang, "Resilient event-triggered output feedback control for load frequency control systems subject to cyber attacks," IEEE Access, vol. 7, pp. 58951-58958, 2019.

[30] X.-M. Zhang, Q.-L. Han, and X. Yu, "Survey on recent advances in networked control systems," IEEE Transactions on Industrial Informatics, vol. 12, no. 5, pp. 1740-1752, 2016.

[31] S. H. Mousavi and H. J. Marquez, "Integral-based event triggering controller design for stochastic LTI systems via convex optimisation," International Journal of Control, vol. 89, no. 7, pp. 1416-1427, 2016.

[32] R. Sakthivel, S. Santra, B. Kaviarasan, and K. Venkatanareshbabu, "Dissipative analysis for networkbased singular systems with non-fragile controller and eventtriggered sampling scheme," Journal of the Franklin Institute, vol. 354, no. 12, pp. 4739-4761, 2017.

[33] M. Shen, S. Yan, Y. Sun, and G. Zhang, "Nonfragile $H_{\infty}$ output feedback control of linear systems with an eventtriggered scheme against unreliable communication links," ISA Transactions, vol. 84, pp. 96-103, 2019.

[34] D. Liu and G.-H. Yang, "Event-triggered non-fragile control for linear systems with actuator saturation and disturbances," Information Sciences, vol. 429, pp. 1-11, 2018.

[35] A. Seuret, F. Gouaisbaut, and Y. Ariba, "Complete quadratic Lyapunov functionals for distributed delay systems," Automatica, vol. 62, pp. 168-176, 2015.

[36] Y. Wang, L. Xie, and C. E. de Souza, "Robust control of a class of uncertain nonlinear systems," Systems \& Control Letters, vol. 19, no. 2, pp. 139-149, 1992.

[37] Y. Zhang, P. Shi, S. K. Nguang, J. Zhang, and H. R. Karimi, "Finite-time boundedness for uncertain discrete neural networks with time-delays and Markovian jumps," Neurocomputing, vol. 140, pp. 1-7, 2014.

[38] Y. Zhang, S. K. Nguang, Y. Song, and P. Shi, "Robust finitetime $H_{\infty}$ control for uncertain discrete-time singular systems with Markovian jumps," IET Control Theory \& Applications, vol. 8, no. 12, pp. 1105-1111, 2014.

[39] E. Tian, Z. Wang, L. Zou, and D. Yue, "Chance-constrained $H_{\infty}$ control for a class of time-varying systems with stochastic nonlinearities: the finite-horizon case," Automatica, vol. 107, pp. 296-305, 2019. 


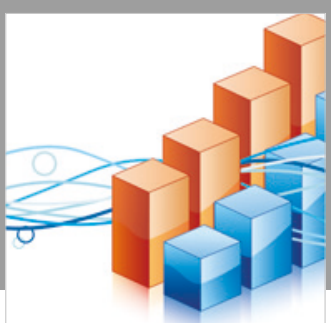

Advances in

Operations Research

\section{-n-m}
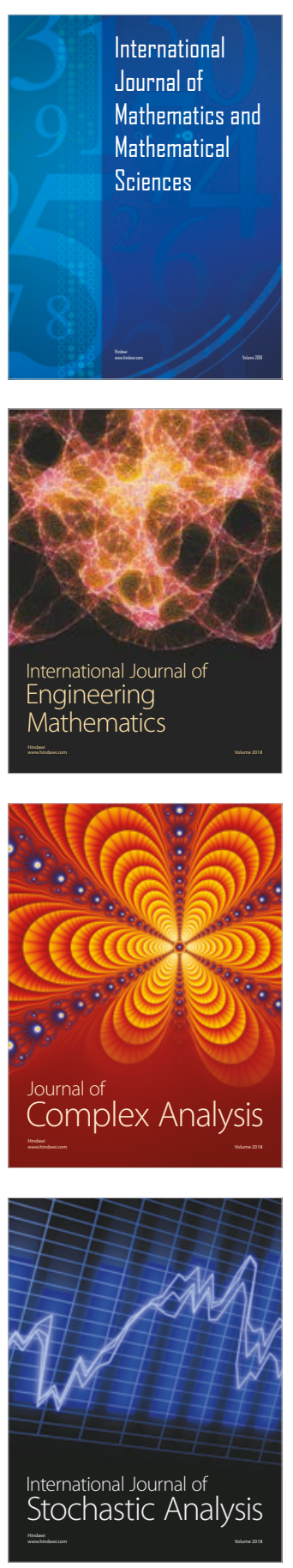
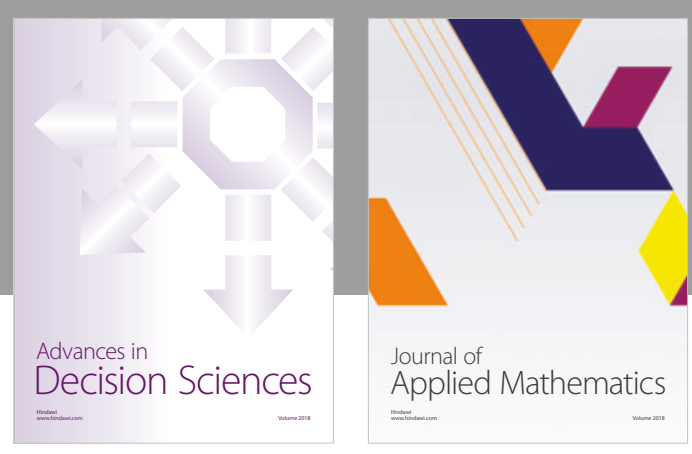

Journal of

Applied Mathematics
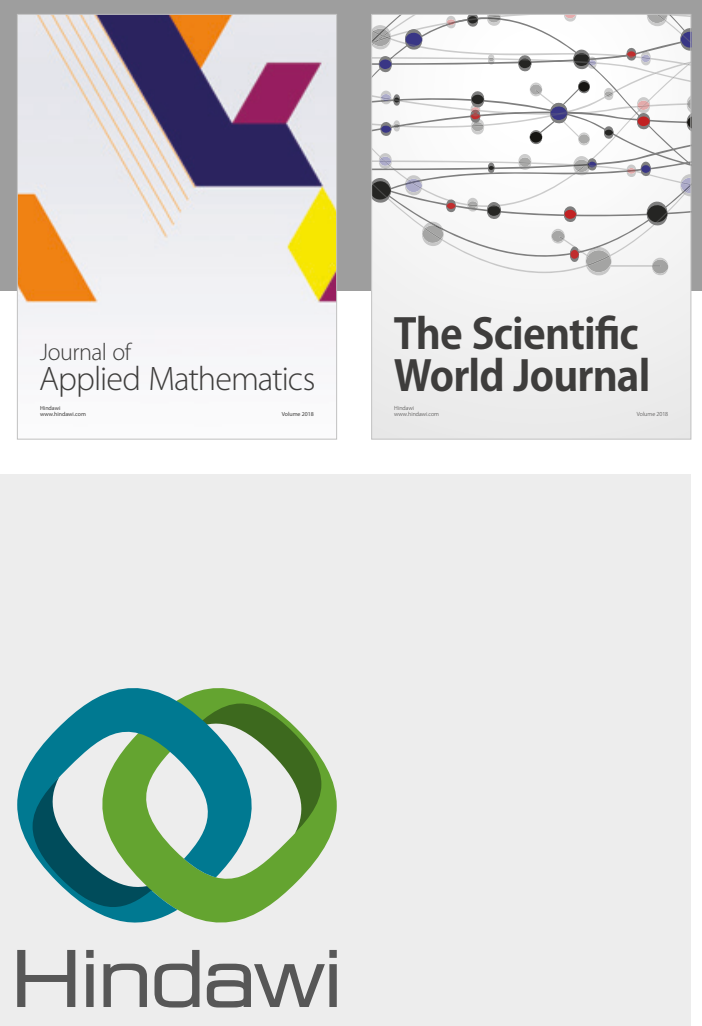

Submit your manuscripts at

www.hindawi.com

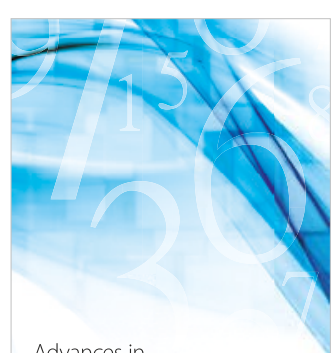

Advances in
Numerical Analysis
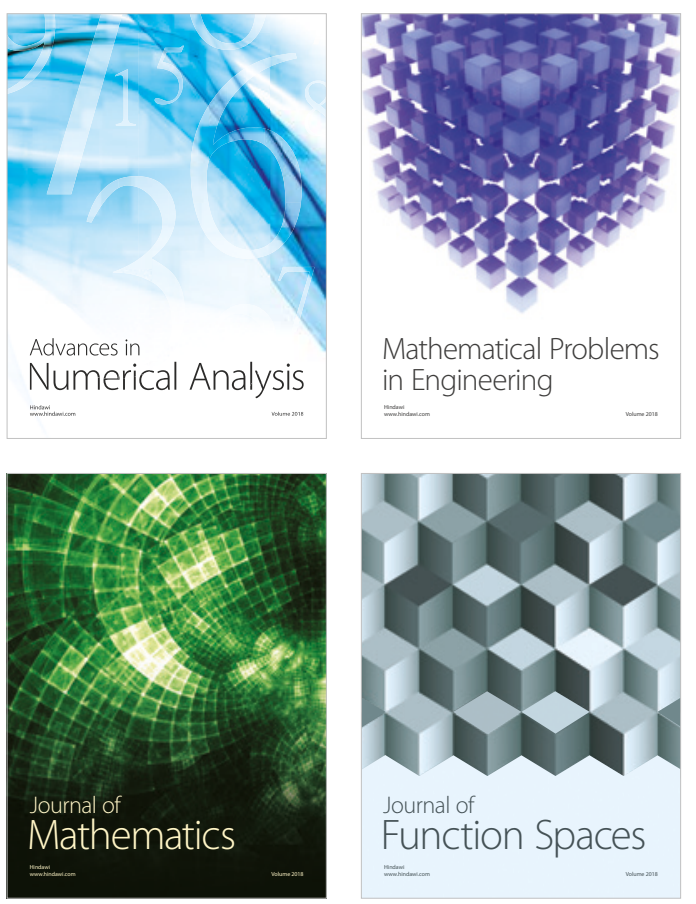

Mathematical Problems in Engineering

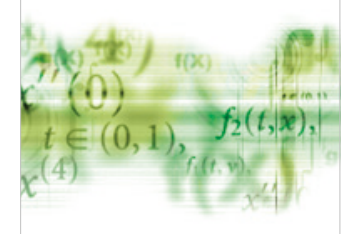

International Journal of

Differential Equations

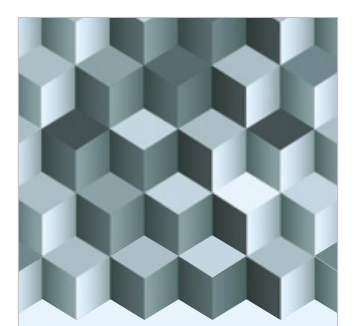

Journal of

Function Spaces
The Scientific

World Journal

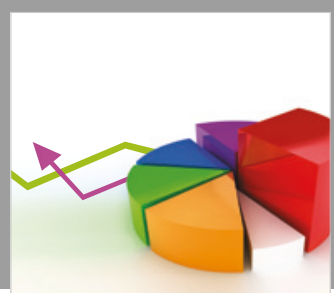

Journal of

Probability and Statistics
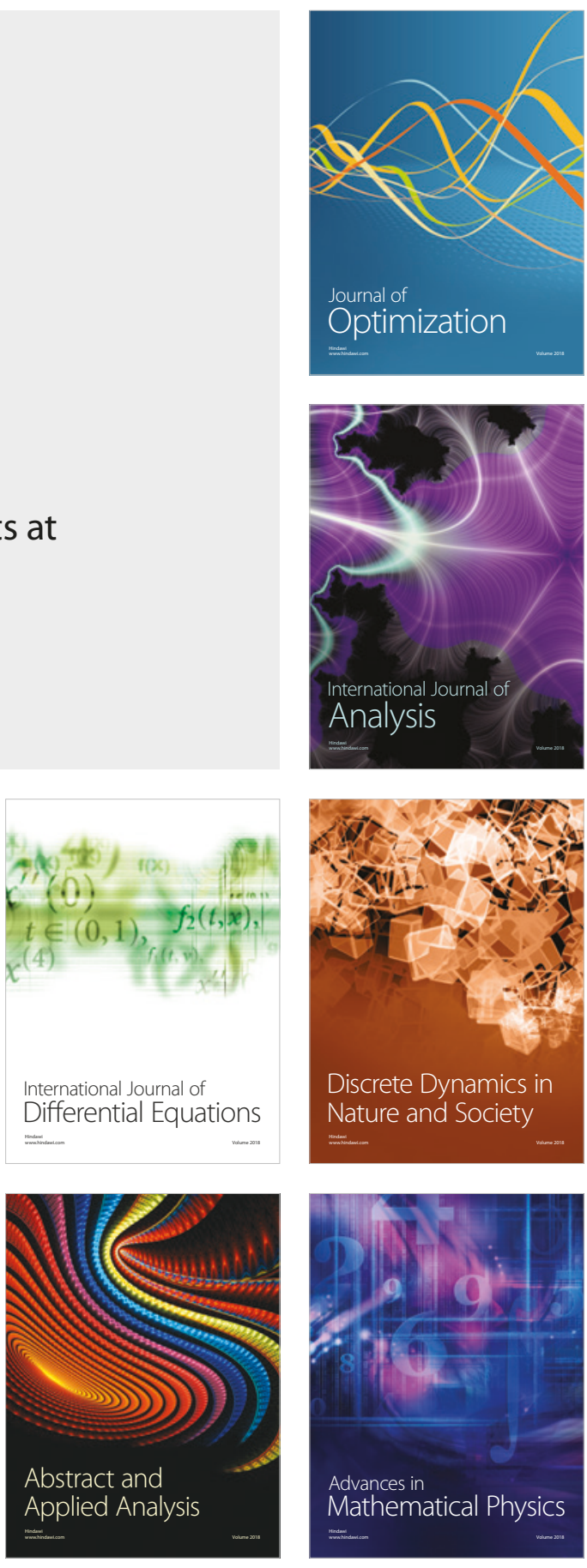